\title{
Global Gravity Field Models from different GOCE Orbit Products
}

J. Schall, A. Shabanloui \& J. Kusche

universitätbonn

Summary

The innovative technique of Satellite Gravity SST observations, point-wise absolute positions of recovery technique is based on Newton's equation Gradiometry enables GOCE to observe small the satellite can be determined. The kinematical of motion, formulated as a boundary value problem gravity field details. Complementary, the lower precise orbit of GOCE, which either is estimated in the form of a Fredholm's type integral equation frequency part of the Earth's gravity field can be with an in-house developed software (Shabanloui, (Mayer-Gürr, 2006). On this poster, the precise orbit extracted based on high-low Satellite to Satellite 2008) or supplied as the precise science orbit by the product of GOCE are compared and the amount of tracking (hl-SST) data, which are observed by the European Space Agency (Bock et al., 2007), is used gravity field information within the different orbit GNSS receiver on-board GOCE. Based on the hl- to recover the Earth's gravity field. The gravity field products is evaluated.

\section{Precise Orbit Determination}

Functional Model: Geometrical high-low GNSS carrier phase observation equations for all observation epochs

Stochastic Model: No correlations for the high-low GNSS GOCE observations

Outlier Detection: Based on the majority voting procedure (Shabanloui, 2008)

Estimation Procedure: Initialization of the GOCE absolute positions based on code pseudo-range observations, improvements of the geometrical GOCE positions, receiver clock offset and ambiguity parameters as float value based on hl-SST carrier phase observations, no dynamical model

Observations: High-low SST of 1 sec. sampling rate within time period $1 / 11 / 2009$ to $11 / 1 / 2010$, GNSS orbits with $15 \mathrm{~min}$. intervals, $30 \mathrm{sec}$. clock offsets, cut-off angle of 15 degree

Geometrical Background Models: All geometrical background models e.g. GNSS antenna offsets, Sagnac effect, GNSS and receiver phase centre offsets and their variations, general \& special relativistic effects

Representation: Point-wise geometrical absolute positions and clock offsets as standard product 3 (sp3) format

\section{Gravity Field Recovery}

Functional Model: Integral equation of Fredholm's type

Stochastic Model: Epoch-wise covariance matrices of the kinematical positions \& consideration of correlations up to the orbit arc length by an estimated empirical covariance function

Outlier Handling: Arc-wise re-weighting of the observation equations

Pseudo Observations: Kinematical precise positions of $1 \mathrm{sec}$. sampling, time period $1 / 11 / 2009$ to $11 / 1 / 2010$, divided in arcs of $30 \mathrm{~min}$ maximum length

Background Models and Disturbing Forces: ITG-Grace2010s, direct tides (from ephemeris), earth tides (IERS2003), ocean tides (EOT08), Dealiasing products (AOD1B), pole tides (IERS2003), non-gravitational forces as measured by the gradiometer (EGG_NOM_1b)

Representation: Spherical harmonic coefficients up to d/o 110, further arcspecific parameter (boundary values, accelerometer offset per axis)

\section{Results from:}

Precise orbits estimated in-an-house (Scenario 1) PSO product (SST PSO 2) as supplied by ESA (Scenario 2)

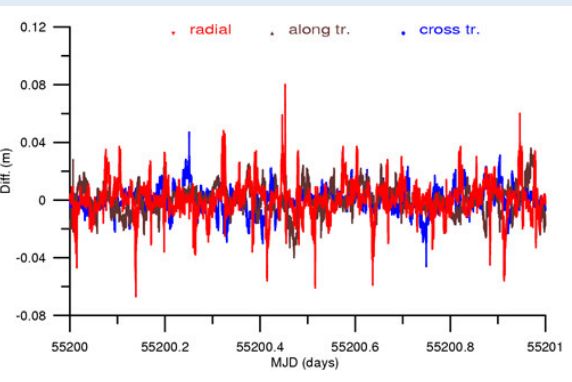

Fig. 1: Differences between estimated orbit and PSO of a sampled day

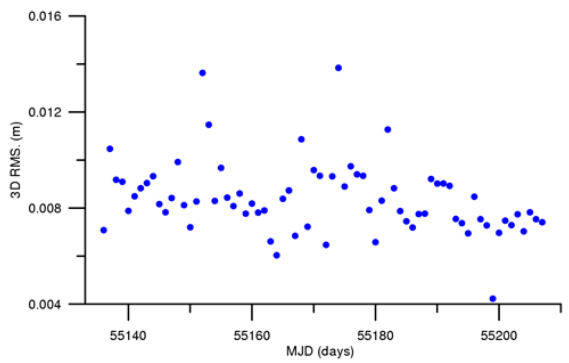

Fig. 2: 3D-RMS of differences between estimated orbit and PSO

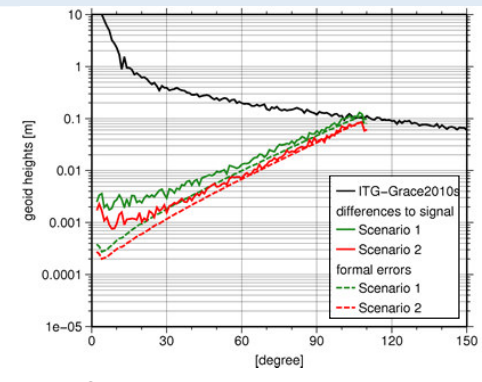

Fig. 3: Comparison of both scenarios, degree variances excl. low order coefficients

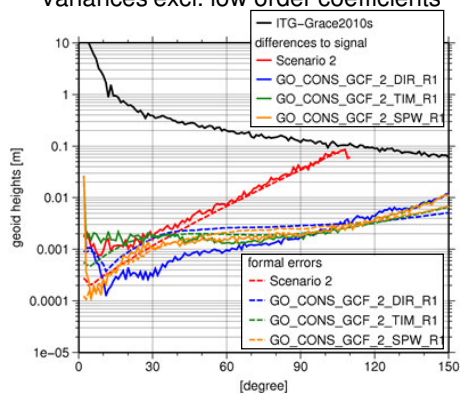

Fig. 4: The scenario 2 GOCE SST model vs. the official ESA combined gravity products

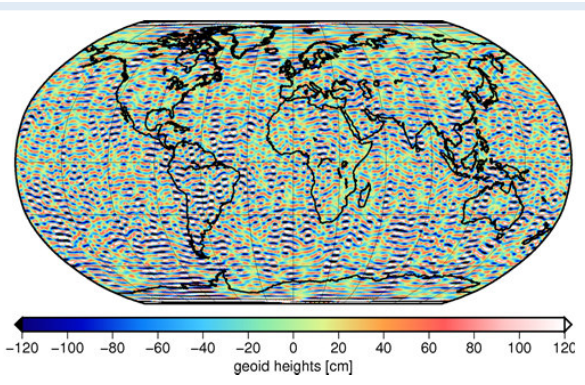

Fig. 5: Geoid heights deviations up to $d / 0110$ of the scenario 1 gravity model from ITG-Grace2010s

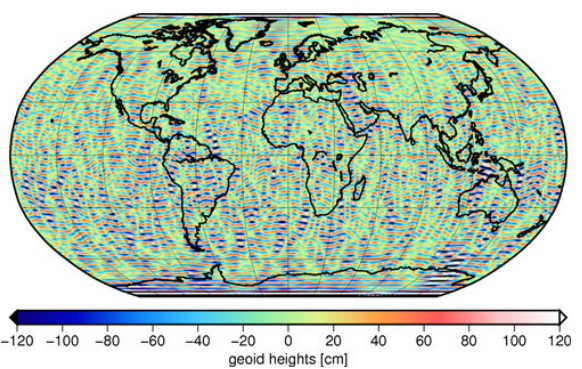

Fig. 6: Geoid heights deviations up to $d / 0110$ of the scenario 2 gravity model from ITG-Grace2010s

\section{Discussion}

The differences between estimated geometrical and officially published PSO orbit of GOCE show good agreement. The differences are in the range of $1-2 \mathrm{~cm}$ (refer to Fig. 1 and 2). The errors of the gravity model calculated from in-an-house processed orbits are a factor of 2-3 larger than when using the official orbit product (refer to Fig. 3, 5 and 6). We expect a better performance of estimated orbits by considering better outlier detection strategies and smaller differences between gravity field solutions by considering the same stochastical information.

Bock, H., Jäggi, A., Svehla, D., Beutler, G., Hugentobler, U., Visser, P. (2007) Precise Orbit Determination for the GOCE Satellite using GPS, J. Adv. Space

Mayer-Gürr, T. (2006) Gravitationsfeldbestimmung aus der Analyse kurzer Bahnbögen am Beispiel der Satellitenmissionen CHAMP und GRACE. Ph.d. thesis, Bonn University, Bonn, Germany.

Shabanloui, A. (2008) A New Approach for a Kinematic-Dynamic Determination of Low Satellite Orbits Based on GNSS Observations. Ph.d. thesis, Bonn University, Bonn, Germany. 